\title{
Notas a la lengua de dos poemas tardobizantinos
}

Some Notes to the Language of Two Late Byzantine Poems

\author{
Juan José Pomer Monferrer * \\ Universitat de València, España \\ juan.pomer@uv.es \\ (iD http://orcid.org/0000-0003-3620-8033
}

\section{Resumen:}

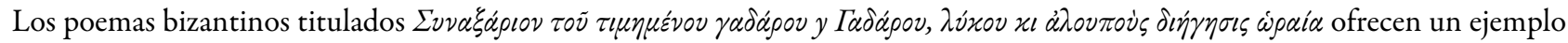
significativo y claro de la interacción entre dos variedades lingüísticas opuestas: de un lado, el griego literario empleado para la composición poética; de otro, el griego popular que iba poco a poco ganando terreno con vistas a reemplazar a la lengua antigua. Palabras ClaVe: Griego literario, Griego popular, Diglosia, Composición poética, Transmisión.

\section{ABSTRACT:}

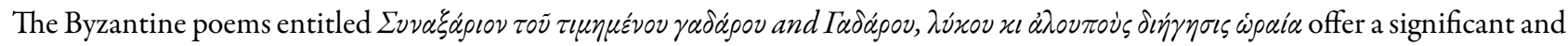
clear example about the interaction between two opposite linguistic registers: from the one hand, the literary Greek used for poetic composition; from the other, the popular Greek which was gradually gaining ground in order to substitute the old language.

KEYWORDS: Literary Greek, Popular Greek, Diglossy, Poetic composition, Transmission.

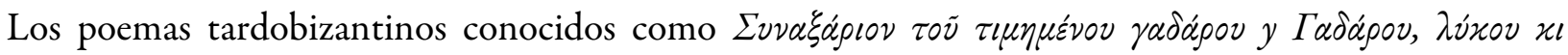

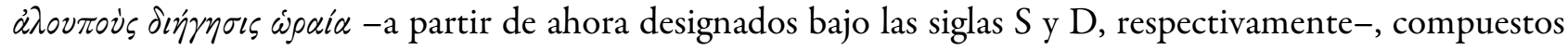
en un patrón métrico muy similar y hacia la misma época, narran una sola historia con escasas diferencias entre ambos, como variantes del que sin duda era un relato bien conocido. ${ }^{1}$ No obstante las evidentes similitudes entre los dos textos, en el plano lingüístico se distinguen muy bien, y a este aspecto se ciñe el presente trabajo. Nuestro objetivo no es otro que el de intentar conocer algo más sobre la diglosia existente en la Grecia de los siglos XIV y XV, cuando más fuerte era, antes aún de la ocupación turca, la separación entre la lengua cancilleresca y litúrgica, por una parte, y la popular por otra.

Los textos que hemos escogido para nuestro estudio pertenecen, por su género y época, a una literatura mal conocida aún, pero que por diversas razones está despertando el interés de muchos investigadores. La edición que hemos utilizado es la clásica de Wagner, aunque también hemos consultado las más recientes de Alexiu y Pöchert. ${ }^{2}$ Los estudios sobre ambos textos, más bien escasos en número, han versado fundamentalmente sobre cuestiones de índole literaria. ${ }^{3}$ Como ya hemos apuntado en un principio, ambos textos presentan un 
sinfín de semejanzas, por lo que se los suele tratar a la vez. ${ }^{4}$ La principal diferencia formal radica en la rima consonante en pareado del poema $\mathrm{D}$. No obstante esta circunstancia, estamos ante sendas composiciones en versos políticos, ${ }^{5}$ el metro narrativo por excelencia de la poesía tardobizantina, por lo que esta variación no tiene mayor trascendencia. Si acaso, desde Krumbacher al menos se ha tendido a considerar que el empleo de la rima en $\mathrm{D}$ supondría una datación más reciente. ${ }^{6}$

La cuestión del origen espacial y temporal de estos poemas dista mucho de estar resuelta siquiera desde el punto de vista metodológico. El propio Krumbacher se limita a recordar el terminus ante quem fijado para cada uno por los respectivos manuscritos, y, en lo que respecta al lugar de composición, señala las Cícladas jonias o Creta en razón de los muchos préstamos italianos registrados, sobre todo en el poema D. ${ }^{7}$ La situación cronológica es tan amplia como el origen geográfico, pues los dos textos pueden remontar a cualquier momento de los siglos XIV y XV. Por consiguiente, en este doble plano nos falta una información más precisa.

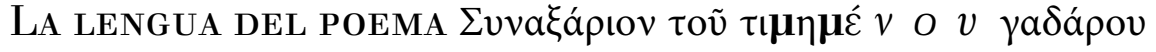

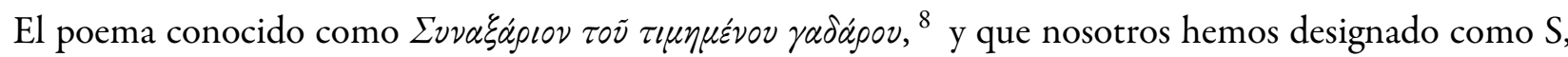
comprende un total de trescientos noventa y tres versos, en los que alternan el discurso directo y la narración, aunque con un claro predominio del primero, que ocupa dos terceras partes del texto. ${ }^{9}$ La lengua no carece de formas arcaizantes, que a menudo conviven con las soluciones propias del griego medieval; así, junto al clásico

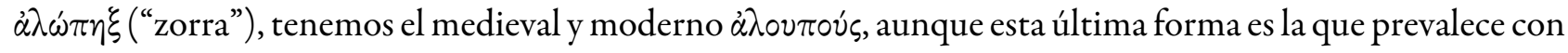
holgada diferencia. ${ }^{10}$ Pero otras veces es la variante clásica la que domina, como en el caso de los adverbios de negación oủk y $\delta \varepsilon ́ v$, de los que es más frecuente el primero. ${ }^{11}$ Es la coexistencia de las dos corrientes, la culta y la popular, la que aquí nos interesa.

Como es de esperar, los dobletes morfológicos son muy abundantes: al lado del clásico $\dot{\eta} \mu \varepsilon \tilde{\iota} \varsigma, \dot{\eta} \mu \tilde{\alpha} \varsigma$, leemos

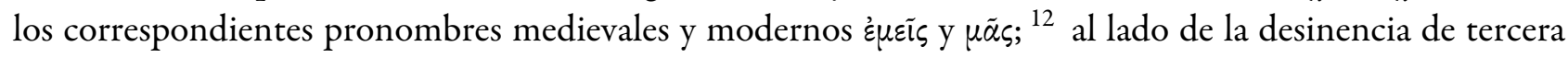

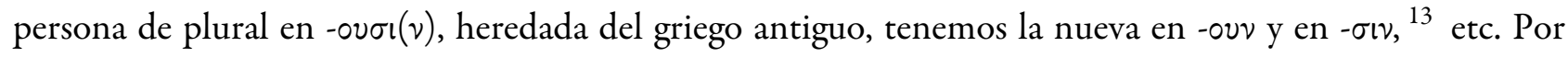
otra parte, si nos fijamos en la selección léxica, anotaremos un número muy bajo de préstamos a lenguas

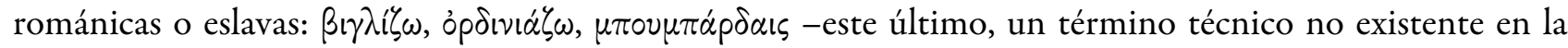
lengua receptora-. ${ }^{14}$ También encontramos algunos vocablos propios de la lengua de la poesía, de claro

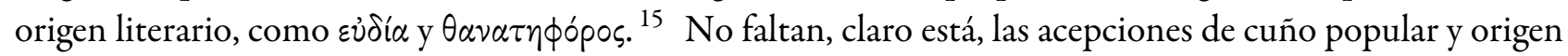
medieval, como $\tau \zeta \eta \mu \alpha ́ \delta$. ${ }^{16}$

El capítulo de la sintaxis nos ofrecerá una más exacta visión del genotipo que subyace a esta lengua poética. Junto a construcciones que solo en el plano literario eran ya posibles, como el empleo de íva como conjunción final, el texto de $S$ nos depara otras nacidas en el griego medieval y destinadas a reemplazar en toda la lengua moderna a sus predecesoras del griego antiguo; valga como ejemplo el giro $\omega \sigma \tau \varepsilon \pi \circ \tilde{\nu} \nu \alpha ́$ para la introducción de una oración consecutiva.

En la sintaxis del sustantivo, anotamos el empleo de tres dativos: dos instrumentales y uno propio. ${ }^{17}$ Este dato por sí solo ya nos sitúa en la tradición de la lengua literaria.

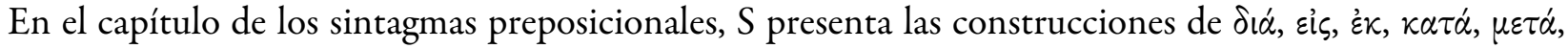

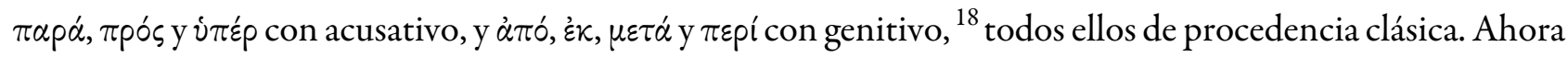
bien, de los muchos ejemplos de $\varepsilon i \varsigma$, una buena parte no son clásicos, bien por razones fonomorfológicas, bien por razones sintácticas. Otros conservan la forma y función propias del griego antiguo, pero se advierte de inmediato que se trata de meras locuciones: es el caso de $\dot{\xi} \xi$ vँ $\pi \nu \circ v$ y $\kappa \alpha \tau \dot{\alpha} \lambda \varepsilon \pi \tau \tau^{\circ} v .{ }^{19}$ 
Otros rasgos de matriz literaria son el empleo de las partículas oป̃v y $\gamma \circ \tilde{v} v$, el de las conjunciones ö $\pi \omega \varsigma$-que además tiene valor completivo-, $\delta$ ı́ $\tau \iota$ y íva y el del infinitivo sustantivado. ${ }^{20}$ Así se completa el conjunto de rasgos de lengua tomados de la tradición, como producto de la formación del autor del texto.

Vamos a ocuparnos ahora de los rasgos propios de la lengua medieval, y carentes, por tanto, de una tradición

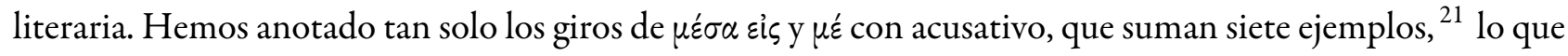
supone un balance más bien escaso. Entre los préstamos destaca en el verso 232 el sustantivo ṕźk $\lambda \alpha$.

Si planteamos un análisis social de los rasgos apuntados, una primera opción pasa por imaginar que uno de los dos registros, el narrativo y el de discurso directo, está más cerca de la lengua popular. Hay argumentos en favor de ambas alternativas, por lo que en un principio procederemos a verificar los datos. La construcción de $\mu \varepsilon ́$ con acusativo, que aparece en cinco ocasiones, solo en una lo hace en un discurso directo. ${ }^{22}$ En cambio, los ejemplos de $\pi \alpha \rho \alpha ́$, $\pi p i ́ v$ y í $\pi \dot{p}$ con acusativo, sendos hápax por cierto, se hallan todos en discurso directo. Pero

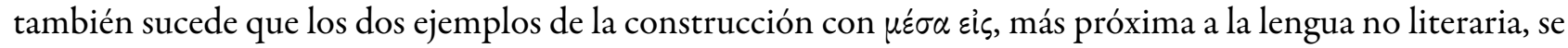
hallan también en discurso directo.

En resumen, en el texto de $S$ no se aprecian diferencias radicales entre los pasajes correspondientes al discurso directo y a la narración. En general, el tipo de lengua que caracteriza al poema se mantiene a lo largo de todo el texto de un modo bastante homogéneo. Ahora bien, no por ello deja de haber algunas variaciones, no por escasas menos significativas. Por un lado, las partes narrativas no presentan rasgos demóticos más acentuados, como habría podido esperarse si el autor hubiera distinguido las intervenciones de los personajes del marco en que se insertan. En cambio, en el discurso directo se registra una mayor tendencia al empleo de arcaísmos, ya que el autor intenta conferir al habla de sus personajes un tono retórico más literario.

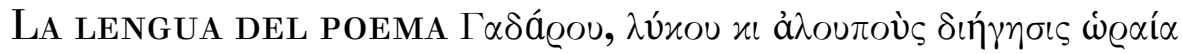

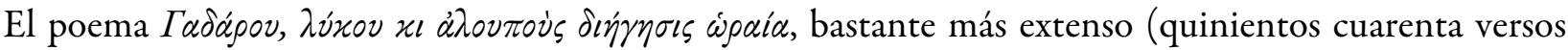
frente a trescientos noventa y tres), presenta en el plano literario numerosas afinidades con el texto que acabamos de analizar. En cambio, en el plano lingüístico hemos observado que los registros correspondientes al tono habitual en ambas obras son muy distintos: mientras $S$ intenta mostrar un nivel de lengua elevado, en D se sitúa muy cerca del habla popular.

En lo que respecta al empleo de préstamos de otras lenguas, ya anotó Krumbacher la mayor preferencia por ellos en D, y en especial por los de origen italiano. ${ }^{23}$ En este grupo hemos de señalar las voces voßź $\lambda \alpha \alpha, \mu \alpha$,

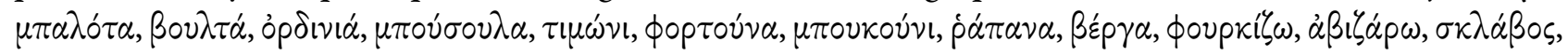

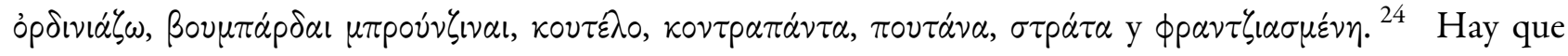

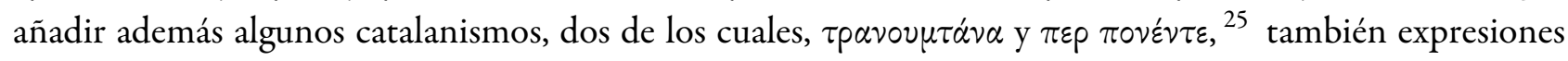
marineras, como buen número de las italianas que acabamos de reseñar, y el tercero un término de carácter más común, $\mu \pi \alpha \rho \alpha ́ k \alpha .^{26}$

En cuanto a la morfología, en $\mathrm{D}$ solo destaca el empleo del adjetivo $\pi \circ \lambda v$ s, de impronta clásica. ${ }^{27} \mathrm{La}$ sintaxis, en cambio, ofrece datos de mayor interés. Así, el conjunto de sintagmas preposicionales de D

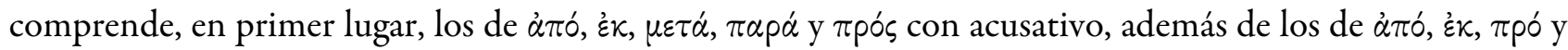
$\mu \varepsilon \tau \dot{\alpha}$ con genitivo. El total de ejemplos es de cuarenta y cuatro, y su distribución muestra que, con la sola excepción de ả đó con acusativo y $\mu \varepsilon \tau \alpha ́$ con genitivo, ninguno de los demás sintagmas pasa de un uso muy restringido. ${ }^{28} \mathrm{Si}$ analizamos la frecuencia de empleo en las partes dialogadas y narradas, hallamos que precisamente $\mu \varepsilon \tau \dot{\alpha}$ con acusativo es mucho más abundante en el discurso directo, que es además donde se

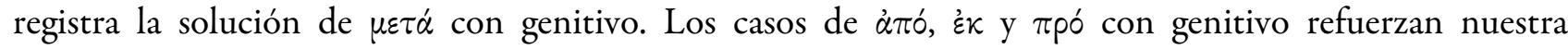
impresión, si bien la muestra no es indicativa por el bajo número de ejemplos. 
Otros sintagmas preposicionales son $\mu \varepsilon^{\prime},{ }^{29} \mu \dot{\varepsilon} \sigma \alpha^{30}$ y $\sigma \dot{\varepsilon}$ con acusativo, ${ }^{31}$ con un total de veintitrés ejemplos. Las hemos consignado aparte por tratarse de construcciones neogriegas, faltas de tradición literaria y más propias, por tanto, de los registros populares y conversacionales.

La sintaxis oracional no destaca por otro rasgo que por el del predominio de la parataxis, ya que las conjunciones de subordinación son más bien escasas en número y parcas en usos: tan solo las causales $\varepsilon^{\prime} \pi \varepsilon^{\prime}{ }^{32}$ y $̇ \pi \varepsilon ı \delta \eta^{33}$ escapan a un registro coloquial y familiar, muy alejado de la lengua más literaria de $S$.

\section{ApUnTes SOBRE LA MÉTRICA DE S y D}

Si bien el objeto del presente trabajo es el análisis de la lengua de estos poemas, creemos conveniente dedicar unas líneas a la métrica de los mismos, en la medida en que tiene también incidencia sobre aquella. Nos limitaremos al recurso del aumento por razones métricas, dado que por medio de las variantes provistas de aumento del tipo silábico se obtiene una sílaba más para el verso. El análisis de $S$ arroja un total de sesenta

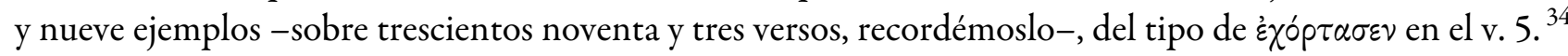
La frecuencia de empleo es de 1 caso por cada 5,7 versos. Para este mismo rasgo en D tenemos ochenta y dos ejemplos -en quinientos cuarenta versos-. ${ }^{35}$ La frecuencia de empleo es de 1 caso por cada 6,6 versos, algo más baja que en el $\Sigma v \nu \alpha \xi \dot{\alpha} \rho \circ \nu$, aunque la diferencia no parece significativa.

Interesa a nuestros propósitos el porcentaje de este recurso en las secciones dialogadas, que en principio deberían estar más próximas a un registro conversacional. En $S$ el aumento silábico se registra en veinticuatro ejemplos sobre sesenta y nueve versos dialogados, lo que arroja una frecuencia de 0,35 ejemplos por verso. En $\mathrm{D}$ se registran cuarenta y seis ejemplos en ochenta y dos versos dialogados, con lo que se obtiene una frecuencia de 0,56 ejemplos por verso. Aquí sí parece haber una diferencia marcada, para cuya exacta valoración pensamos que se requiere un corpus más amplio de ejemplos.

\section{Conclusiones}

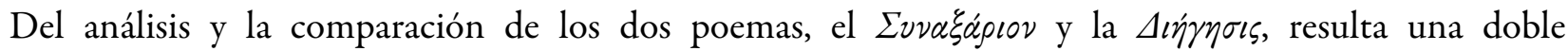
constatación: por una parte, la lengua utilizada en ambos, que es la de la lírica tardobizantina, presenta unas características comunes, que derivan de la coexistencia de formas antiguas y modernas con predominio de estas últimas, mientras que en la prosa contemporánea, más próxima en general a los usos de la lengua diaria, se acentúa aún más el empleo de las soluciones demóticas; por otra, y aun tratándose en el fondo de una sola obra -de un único metatexto, si se nos permite la expresión-, el color dialectal de una y otra versión permite distinguirlas con claridad, pues $S$ tiende a utilizar los registros más literarios, y $\mathrm{D}$ los menos literarios.

Creemos de gran interés el contraste de ambos textos como una fuente más para el conocimiento de la diglosia de la cultura tardobizantina.

\section{REFERENCIAS}

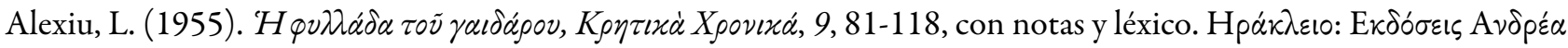
Г. Kàokalpivoú.

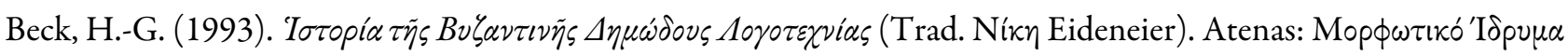

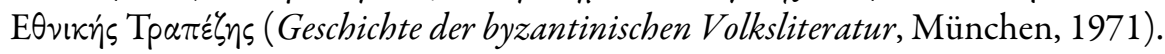

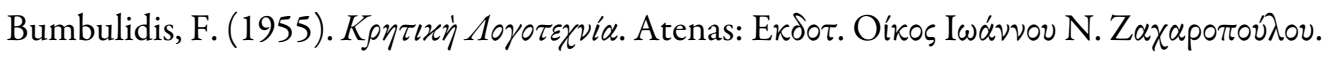

Jeffreys, M. J. (1974). The Nature and Origins of the Political Verse. Dumbarton Oaks Papers, 28,141-195. 


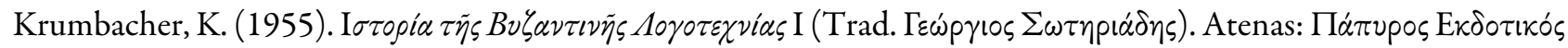

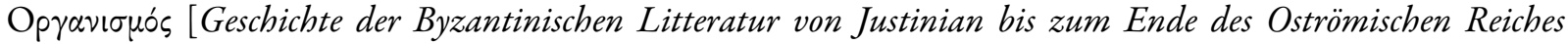
(527-1453), München, 1897, $2^{\mathrm{a}}$ ed.].

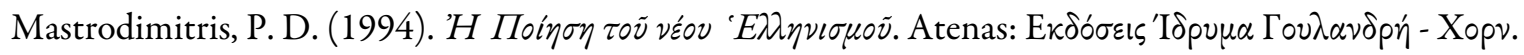

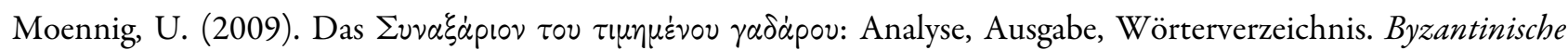
Zeitschrift, 102, 109-166.

Pochert, C. (1991). Die Reimbildungin der spät- und postbyzantinischen Volksliteratur. En N. M. Panagiotakis (Ed.), Neograeca Medii Aevi (pp. 45-131). Colonia: Romiosini.

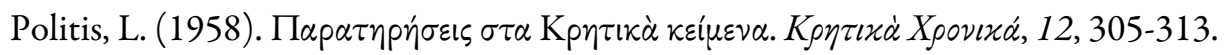

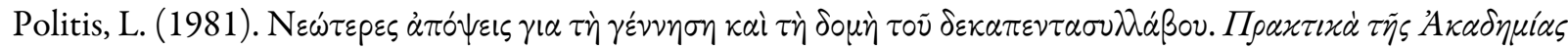
$A \theta \eta \nu \tilde{\omega} \nu, 56,211-228$.

Redondo, J. (1999). Hel.lenismes al català medieval: bilingüisme o llengua franca? En M. Aleza, M. Fuster \& B. Lépinette (Eds.), Quaderns de Filologia. Estudis Lingüistics IV. El contacto lingüistico en el desarrollo de las lenguas occidentales (pp. 173-186). València: Universitat de València.

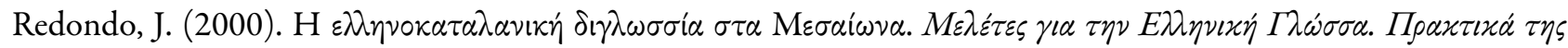

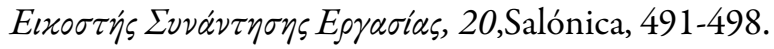

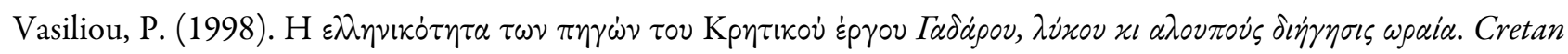
Studies, 6, 267-285.

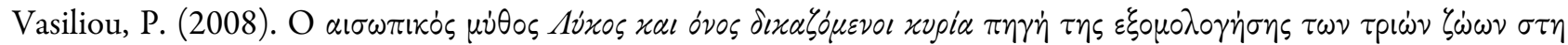

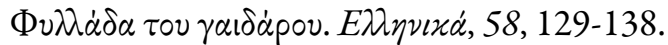

Wagner, W. (Ed.). (1874). Carmina Graeca Medii Aevi [pp. 112-123 (S) y 124-140 (D)]. Leipzig: Teubner.

\section{Notas}

1 No compartimos la extrema prevención de Beck (1993, p. 277) cuando sobre el origen de ambos textos afirma: ${ }^{\mathrm{H}} \chi^{\varepsilon} \dot{\sigma} \eta$

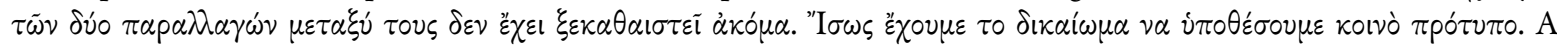
nuestro modo de ver, no existe otra opción para explicar los paralelismos entre ambos poemas. Sobre las fuentes del poema original, véase Vasiliou (1998 y 2008).

2 Wagner (1874); Alexiu (1955); Pochert (1991). Véase también Politis (1958).

3 Cf. Bumbulidis (1955, pp.59-63); Beck (1993, pp. 275-277).

4 Cf. Krumbacher (1955, pp. 892-895).

5 Para este patrón métrico, véase Jeffreys (1974), que incide en el origen popular. Politis (1981) ha formulado una interesante aportación relativa al papel de los poetas profesionales en la configuración y difusión del metro.

6 Krumbacher (1955, p. 895). La tesis es recogida por Mastrodimitris (1994, p. 351).

7 Krumbacher, ibid.

8 Para una edición reciente véase Moennig (2009).

9 Las secciones narrativas -a veces reducidas a un hemistiquio- ocupan los versos 1-21, 42-48, 86-96, 112-116, 123-126, 136-141, 208-212, 221-222, 224-225, 250-253, 260-261, 265, 286-287, 292-294, 295, 304-323, 333, 359, 372-379 y 385-394, lo que suma un total de 110 vv., aproximadamente. El discurso directo, mucho más extenso, ocupa los versos 22-41, 49-85, 97-111, 117-122, 126-135, 142-297, 212-220, 222-223, 226-249, 254-259, 262-264, 266-285, 288-291, 294, 295-303, 323-332, 334-358, 360-371 y 379-384, con un total de 293 vv., aproximadamente.

10 Registramos la forma antigua $\dot{\alpha} \lambda \omega \dot{\pi} \pi \eta \xi$ en los vv. 45, 114 y 265, y la variante moderna $2 \lambda \lambda$ ov $\pi \circ v_{s}$ en los vv. 10, 48, 96, 136, $252,286,292,318,319,322,333,359$ y 380.

11 Se registra oủk en los vv. 4, 45, 63, 87, 161, 231, 259 (bis), 328, 370 y 387, y dév en los vv. 4, 23, 355, 370 y 392.

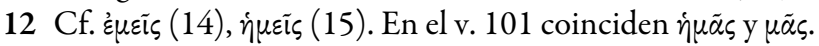

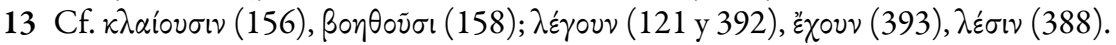

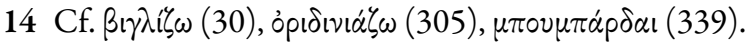

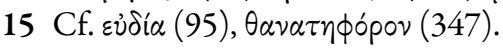

16 Cf. v. 130.

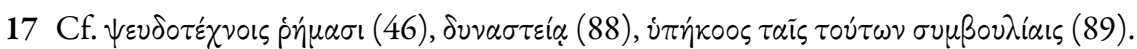




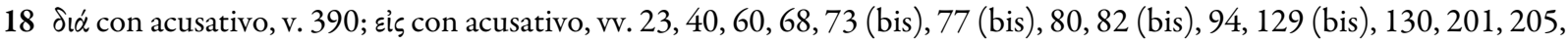

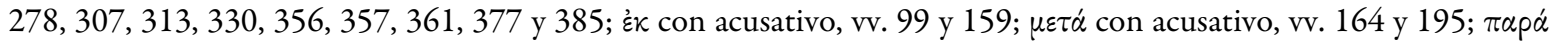

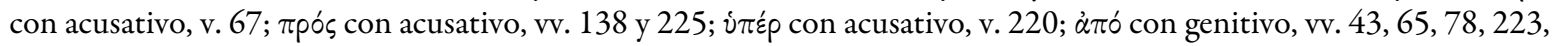

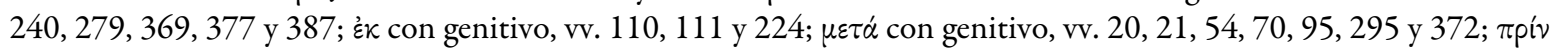
con genitivo, v. 276; $\pi \varepsilon p i ́$ con genitivo, v. 207.

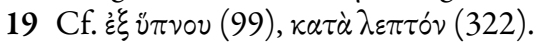

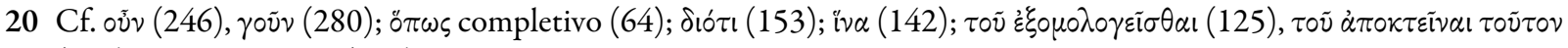
(267) у $\tau \circ \tilde{v} \theta \alpha \nu \alpha \tau \tilde{\omega} \sigma \alpha l(366)$.

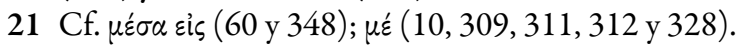

22 Cf. v. 328.

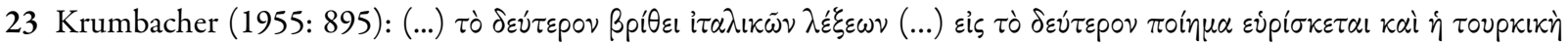

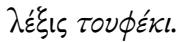

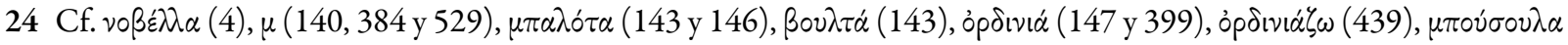

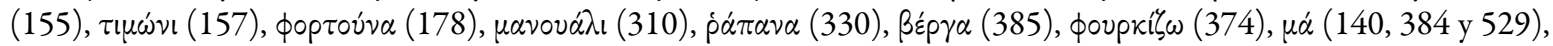

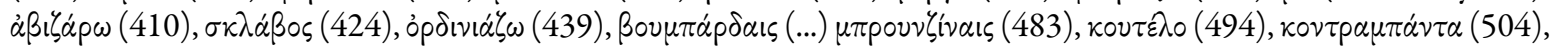

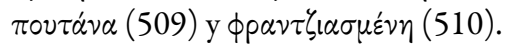

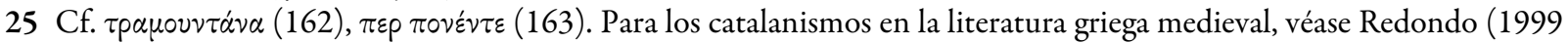
y 2000).

26 Cf. $\mu \pi \alpha \rho \dot{\alpha} \kappa \alpha$ (253).

27 Cf. v. 452.

28 Se registra ḋंó con acusativo en los vv. 19, 28, 30, 102, 188, 195, 200, 285, 286, 296, 308, 314, 318, 387, 452, 462, 490, 528 y 540; '́k con acusativo, vv. 20, 469, 476 y 512; $\mu \varepsilon \tau \dot{\alpha}$ con acusativo, vv. 12, 58, 62, 120, 121, 124, 228, 398 y 501 ; $\pi \alpha \rho \alpha ́$ con acusativo, v. 255; $\pi$ pós con acusativo, vv. 212, 298, 304 y 326, aunque el ejemplo del v. 304 corresponde a una

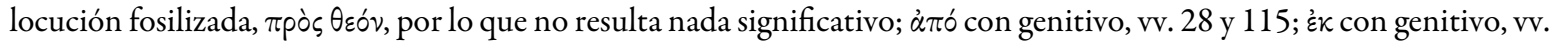
106, 387 y 401, aunque el ejemplo del v. 106 corresponde a otra locución, '̇́ $\sigma \tau \dot{\eta} \theta 0 v$, que nosotros creemos fosilizada; $\pi$ pó con genitivo, v. 179; $\mu \varepsilon \tau \dot{\alpha}$ con genitivo, vv. 418 y 511.

29 Cf. vv. $47-48$ (ter), 56, 239, 242, 385, 342, 353, 363, 407, 443, 458, 533-534 (ter) y 539.

30 Cf. vv. 132 y 139.

31 Cf. vv. 128 (ter) y 368.

32 Cf. v. 380.

33 Cf. v. 111.

34 Cf. vv. 4, 18, 19, 26, 36, 48, 84, 89, 92, 100, 110, 112, 137 (bis), 138, 152, 153, 161, 163, 165, 169, 186, 188, 190, 191, 192, 210, 211, 213 (bis), 220, 221, 224, 226, 229 (bis), 230, 231, 233, 234, 241, 244, 262, 270, 272, 293, 304, 305, 307 , 311,312 (bis), 313, 320, 328, 346, 348, 349 (bis), 352, 353, 356, 366, 373, 379, 380, 382, 383 у 386.

35 Cf. vv. 3, 9, 11, 13, 187, 23, 35, 39, 47, 57, 61, 141 (bis), 142, 152, 173, 174, 177, 178, 186 (bis), 187, 195, 209 (bis), 210, 228, 237, 238, 251, 252, 253, 255, 276, 281, 282, 283, 285, 288, 291, 292 (bis), 305 (bis), 311, 312, 313, 326, 332, 333, 334, 368, 375, 406, 411, 428, 429, 435, 437, 460, 461, 463, 464, 465, 466, 467, 485, 488 (bis), 493, 495 (bis), 498 (bis), $501,503,512,516,527,531,532$ y 539 .

\section{NOTAS DE AUTOR}

* Juan José Pomer Monferrer es licenciado en Filología Clásica (1994) y en Humanidades (2010) por la Universitat de València y doctor en Filología Clásica (2015) por la misma institución, con la tesis titulada Aticisme i koinéen els llibres I-III de les Etiòpiques d'Heliodor, con Mención Internacional y calificada con Excelente Cum Laude. Es profesor de Griego y Cultura Clásica en centros de Enseñanza Secundaria (desde 1995) y de Filología Griega en la Universitat de València. Ha publicado trabajos en revistas y en libros de carácter internacional y ha participado en la edición de numerosos libros científicos y en ponencias y comunicaciones en encuentros internacionales, cuyos temas tratan las líneas de investigación del interesado: la novela griega antigua, especialmente el autor Heliodoro de Émesa; la historia de la lengua griega, incluido el griego moderno; la literatura griega de época imperial y la recepción de la literatura clásica en literaturas hispánicas. Entre sus publicaciones se encuentran los artículos "Herois clàssics i herois cavallerescos en espais marítims i espais ficcionals" (2018) y "Focs que no cremen en l'hagiografia tardoantiga i bizantina" (2018), el capítulo

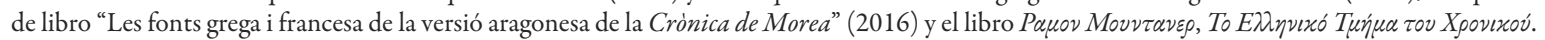
Ramon Muntaner, La secció grega de la Crónica (2016, en coautoría). Ha participado en la organización de Jornadas dedicadas al mundo clásico y en grupos de investigación. Ha realizado estancias en diversas universidades griegas: Atenas, Tesalónica, Patras, Creta. 